\title{
Magnetars are super hot and super cool
}

\author{
Wynn C. G. Ho ${ }^{1}$, Kostas Glampedakis ${ }^{2}$, and Nils Andersson ${ }^{1}$ \\ ${ }^{1}$ School of Mathematics, University of Southampton, Southampton, SO17 1BJ, UK \\ email: wynnho@slac.stanford.edu \\ ${ }^{2}$ Departamento de Física, Universidad de Murcia, E-30100 Murcia, Spain
}

\begin{abstract}
We examine to what extent the inferred surface temperature of magnetars in quiescence can constrain the presence of a superfluid in the neutron star core and the role of magnetic field decay in the core. By performing detailed simulations of neutron star cooling, we show that extremely strong heating from field decay in the core cannot produce the high observed surface temperatures nor delay the onset of neutron superfluidity in the core. We find that it is not possible to conclude that magnetar cores are in a non-superfluid state purely from high surface temperatures. We find that neutron superfluidity in the core occurs less than a few hundred years after neutron star formation for core fields $<10^{16} \mathrm{G}$. Thus all known neutron stars, including magnetars, without a core containing exotic particles, should have a core of superfluid neutrons and superconducting protons.
\end{abstract}

Keywords. dense matter, neutrinos, pulsars: general, stars: evolution, stars: neutron

Anomalous X-ray pulsars and soft gamma-ray repeaters form the magnetar class of neutron stars, i.e., neutron stars which possess superstrong magnetic fields $\left(B \gtrsim 10^{14} \mathrm{G}\right)$ in most cases. Their strong fields likely power the activity seen in these objects (see Woods \& Thompson 2006; Mereghetti 2008, for review). One notable property of magnetars is that their observed surface temperatures in quiescence are significantly higher than those of other neutron stars of a similar age (see Fig. 1). In fact, they are too high for neutron stars that cool passively, i.e., without an additional source of internal heat (accretion heating can be excluded by, e.g., non-detections of binary companion or disk emission). An interesting problem concerns the heat generated from magnetic field decay, which has been proposed to be the source for the high temperatures of magnetars (see, e.g., Thompson \& Duncan 1996). This heat can strongly influence the time/age at which the core becomes superfluid if heating/field decay occurs in the core (Thompson \& Duncan 1996; Arras et al. 2004; Dall'Osso et al. 2009). The problem is important since the presence of superfluid components has a strong impact on magnetar interior dynamics.

Fig. 2 shows the temperature as a function of density and age for a neutron star cooling model that has no additional sources of internal heat (left panel), core heating due to magnetic field decay (center panel), and crust heating (right panel); see Ho et al. (2012) for details on the heat source. At very early ages, the neutron star core cools so rapidly by neutrino emission that the crust does not have time to react; thus the crust is hotter than the core. A cooling wave travels from the core to the surface, bringing the NS to a relaxed, isothermal state. Depending on the properties of the crust, the relaxation time is $\sim 10-100 \mathrm{yr}$ (Lattimer et al. 1994; Gnedin et al. 2001; Yakovlev et al. 2011). The center panel shows that the extra heat generated from magnetic field decay in the core is efficiently removed by neutrino emission; the surface temperatures from this (core heating only) model is too low to explain the observed temperatures of magnetars (see Fig. 1). Meanwhile, the right panel shows that a heat source in the outer crust can very effectively maintain a high temperature near the surface (see Fig. 1) and can power magnetar surface emission (see also Kaminker et al. 2006, 2009). 


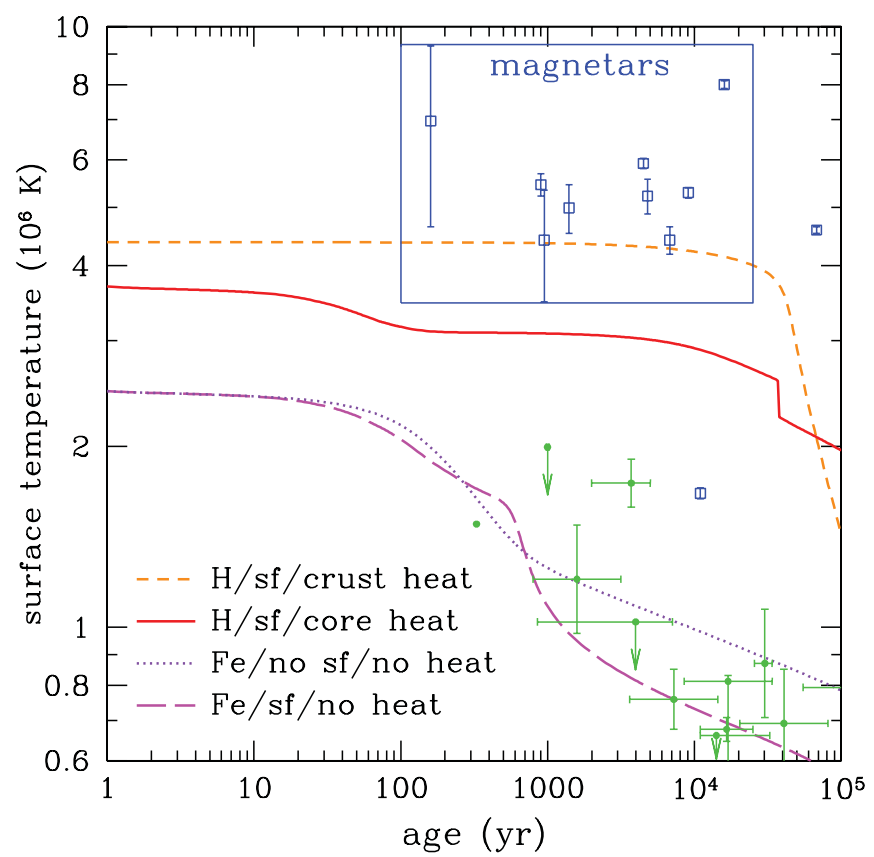

Figure 1. Surface temperature evolution for models with superfluid and crust heating (shortdashed), superfluid and core heating (solid), no superfluid and no heating (dotted), and superfluid and no heating (long-dashed); models with heating have a fully accreted hydrogen envelope (with a $10^{15} \mathrm{G}$ radial surface magnetic field), while models with no heating have an iron envelope. Initial core magnetic field $=10^{16} \mathrm{G}$ and heating/field-decay time-scale $=10^{4} \mathrm{yr}$. Data points are magnetars and other neutron stars taken from the McGill SGR/AXP Online Catalog and those listed in Chevalier (2005); Yakovlev et al. (2008); Ho \& Heinke (2009); Kaminker et al. (2009), respectively.

Fig. 2 also shows the critical temperatures for the onset of superfluidity of core protons in the singlet state $\left(T_{\mathrm{cp}}\right)$, core neutrons in the triplet state $\left(T_{\mathrm{cnt}}\right)$, and crust neutrons in the singlet state $\left(T_{\text {cns }}\right)$; note that the models for the superfluid critical temperature in the core are obtained from fitting the rapid cooling seen in the Cassiopeia A neutron star (Heinke \& Ho 2010; Page et al. 2011; Shternin et al. 2011). We can thus see the two main effects of superfluidity on neutron star cooling: slower cooling in the core after protons become superconducting and faster cooling after neutrons become superfluid due to neutrino emission from Cooper pair formation; the latter is strongest in regions near the critical temperature (see Yakovlev \& Pethick 2004; Page et al. 2006, for review). It is also evident that most of the core becomes superconducting after $\sim 1 \mathrm{yr}$, regardless of the presence of additional heating. For neutrons in the core and heating in the crust, effective thermal decoupling between outer crust and core means that the core cools as if there is no additional source of heat (compare right and left panels of Fig. 2) and the core temperature drops below the critical temperature for neutron superfluidity after a few hundred years. On the other hand, the center panel shows that with extreme core heating (with magnetic field decay time-scale $=10^{4} \mathrm{yr}$ and initial field $=10^{16} \mathrm{G}$ ), the core temperature stays above $T_{\text {cnt }}$ for $\gg 10^{2}$ yr. However, by more properly accounting for the superconducting state of core protons, the decay time-scale can be $\gg 10^{4} \mathrm{yr}$ (Glampedakis et al. 2011; Ho et al. 2012). This reduces the heating rate, since the rate is inversely proportional to decay time-scale, and renders core heating ineffective against cooling by strong neutrino emission. As a result, the onset of core neutron superfluidity 

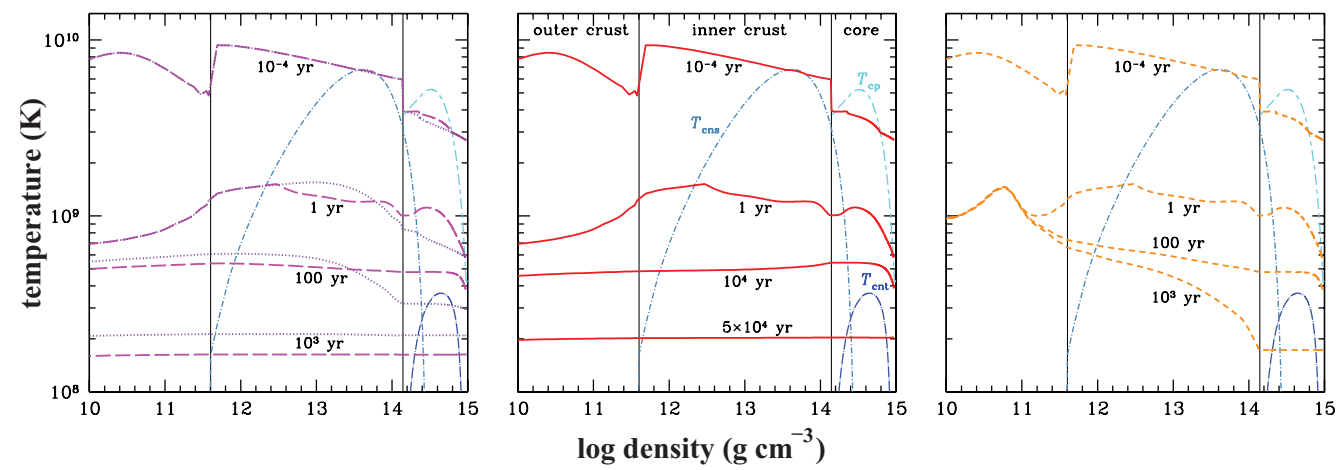

Figure 2. Neutron star interior temperatures. Left: Cooling models with/without superfluidity (dashed/dotted) and no heating. Center: Model with superfluidity and normal core heating (see text). Right: Model with superfluidity and crust heating. Initial magnetic field $=10^{16} \mathrm{G}$ and heating/field-decay time-scale $=10^{4}$ yr. Critical temperatures for neutron singlet $\left(T_{\mathrm{cns}}\right.$; dot-short-dashed), neutron triplet $\left(T_{\mathrm{cnt}}\right.$; dot-long-dashed), and proton singlet $\left(T_{\mathrm{cp}}\right.$; short-longdashed) are shown. Vertical lines indicate boundaries between core and inner crust and inner and outer crusts.

is not delayed and occurs after a few hundred years. Thus the core of all neutron stars can be treated as being in a superfluid and superconducting state after the neutron star is a few hundred years old. Further details can be found in Ho et al. (2012).

WCGH and NA acknowledge support from the RAS and STFC in the UK. KG is supported by the Ramón y Cajal Programme in Spain.

\section{References}

Arras, P., Cumming, A., \& Thompson, C. 2004, ApJ, 608, L49

Chevalier, R. A. 2005, ApJ, 619, 839

Dall'Osso, S., Shore, S. N., \& Stella, L. 2009, MNRAS, 398, 1869

Glampedakis, K., Jones, D. I., \& Samuelsson, L. 2011, MNRAS, 413, 2021

Gnedin, O. Y., Yakovlev, D. G., \& Potekhin, A. Y. 2001, MNRAS, 324, 725

Heinke, C. O. \& Ho, W. C. G. 2010, ApJ, 719, L167

Ho, W. C. G. \& Heinke, C. O. 2009, Nature, 462, 71

Ho, W. C. G., Glampedakis, K., \& Andersson, N. 2012, MNRAS, 422, 2632 [erratum: 425, 1600]

Kaminker, A. D., et al. 2006, MNRAS, 371, 477

Kaminker, A. D., et al. 2009, MNRAS, 395, 2257

Lattimer, J. M., et al. 1994, ApJ, 425, 802

Mereghetti, S. 2008, A\&A Rev., 15, 225

Page, D., Geppert, U., \& Weber, F. 2006, Nucl. Phys. A, 777, 497

Page, D., et al. 2011, Phys. Rev. Lett., 106, 081101

Shternin, P. S., et al. 2011, MNRAS, 412, L108

Thompson, C. \& Duncan, R. C. 1996, ApJ, 473, 322

Woods, P. M. \& Thompson, C. 2006, in: W. H. G. Lewin \& M. van der Klis (eds.), Compact Stellar X-ray Sources (Cambridge: Cambridge University Press), p. 547

Yakovlev, D. G. \& Pethick, C. J. 2004, ARA\& A, 42, 169

Yakovlev, D. G., et al. 2008, in: C. G. Bassa, Z. Wang, A. Cumming, \& V. M. Kaspi (eds.), AIP Conf. Proc. Vol. 983, 40 Years of Pulsars (Melville: American Inst. Phys.), p. 379

Yakovlev, D. G., et al. 2011, MNRAS, 411, 1977 